\title{
Field dependent quasiparticles in a strongly correlated local system II
}

\author{
J. Bauer and A.C. Hewson \\ Department of Mathematics, Imperial College, London SW7 2AZ, United Kingdom
}

(Dated: November 13, 2018)

\begin{abstract}
We extend the renormalized quasiparticle description of the symmetric Anderson model in a magnetic field $H$, developed in earlier work, to the non-symmetric model. The renormalized parameters are deduced from the low energy NRG fixed point for arbitrary field values. We find quasiparticle resonance widths, $\tilde{\Delta}_{\sigma}(H)$, which depend in general on the spin $\sigma$ as well as $H$. The low temperature static properties can be expressed completely in terms of these parameters, which can also be used as inputs for a renormalized perturbation theory. We show that taking into account repeated quasiparticle scattering gives results for the longitudinal and transverse dynamic spin susceptibilities which are in very good agreement with those obtained from direct NRG calculations.

PACS numbers: $72.15 . \mathrm{Qm}, 75.20 \mathrm{Hr}, 73.21 . \mathrm{La}$
\end{abstract}

\section{INTRODUCTION}

In an earlier paper ${ }^{\underline{1}}$ (hereafter referred to as I), we showed how the low energy behavior of the particle-hole symmetric Anderson impurity mode ${ }^{2}$ can be described in terms of field-dependent quasiparticles. This model is characterized by the three independent parameters, $\varepsilon_{d}$, the impurity level, $\Delta$, the broadening of this level due to the hybridization with conduction electrons, and $U$, the interaction at the impurity site. In the absence of a magnetic field, it was shown earlier ${ }^{3,4,5}$ that the low energy behavior can be described by an effective version of the same model with three corresponding renormalized parameters, $\tilde{\varepsilon}_{d}, \tilde{\Delta}$, and $\tilde{U}$. Subsequently, this approach was extended to include a magnetic field $H$, and the parameters were then found to be field-dependent, $\tilde{\varepsilon}_{d, \sigma}(H), \tilde{\Delta}_{\sigma}(H)$, and $\tilde{U}(H)$. One way to calculate these parameters is from the low energy excitations of the numerical renormalization group (NRG) fixed point which was used in the earlier paper I. A strong magnetic field tends to freeze the spin fluctuations and leads to a derenormalization of the quasiparticles. On increasing the field from zero in the strong coupling case the parameters for the quasiparticles slowly revert to their uncorrelated mean field values in the extreme high field limit.

The renormalized parameters are not just a convenient way of describing the low energy behavior; they completely specify the model. A renormalized perturbation theory (RPT) can be set up in which the free propagators correspond to fully dressed quasiparticles 6.7 . This formalism is particularly effective for describing the Fermi liquid regime, as only diagrams up to second order have to be taken into account to obtain asymptotically exact results for the $T=0$ susceptibilities, and the leading $T^{2}$ term in the conductivity (I). This perturbation expansion is not restricted to the low energy and low temperature regime, and can be used for calculations on all energy scales. We have shown that a very good description of the $T=0$ spin and charge dynamics for the Anderson model in the Kondo regime can be obtained by summing the RPT diagrams for repeated quasiparticle scattering ${ }^{8}$. The results give an accurate description of the spin and charge susceptibilities for arbitrary magnetic field values $H$, and for frequencies $\omega$ extending over a range significantly larger than the Kondo temperature $T_{\mathrm{K}}$. The Korringa-Shiba relation ${ }^{9}$ and the sum rules for the spectral density are satisfied.

In this paper we show that this approach can be extended to the non-symmetric Anderson model. There are significant differences in this case, as the parameters acquire a spin dependence, and formulae given earlier have to be generalized.

\section{THE NON-SYMMETRIC ANDERSON MODEL IN A MAGNETIC FIELD}

The Hamiltonian for the Anderson model ${ }^{2}$ is

$$
\begin{aligned}
& H_{\mathrm{AM}}=\sum_{\sigma} \varepsilon_{\mathrm{d}, \sigma} d_{\sigma}^{\dagger} d_{\sigma}+U n_{\mathrm{d}, \uparrow} n_{\mathrm{d}, \downarrow} \\
& +\sum_{k, \sigma}\left(V_{k, \sigma} d_{\sigma}^{\dagger} c_{k, \sigma}+V_{k, \sigma}^{*} c_{k, \sigma}^{\dagger} d_{\sigma}\right)+\sum_{k, \sigma} \varepsilon_{k, \sigma} c_{k, \sigma}^{\dagger} c_{k, \sigma},
\end{aligned}
$$

where $\varepsilon_{\mathrm{d}, \sigma}=\varepsilon_{\mathrm{d}}-\sigma g \mu_{\mathrm{B}} H / 2$ is the energy of the localized level at an impurity site in a magnetic field $H, U$ the interaction at this local site, and $V_{k, \sigma}$ the hybridization matrix element to a band of conduction electrons of spin $\sigma$ with energy $\varepsilon_{k, \sigma}-\sigma g_{c} \mu_{\mathrm{B}} H / 2$, where $g_{c}$ is the g-factor for the conduction electrons. When $U=0$ the local level broadens into a resonance, corresponding to a localized quasi-bound state, whose width depends on the quantity $\Delta_{\sigma}(\omega)=\pi \sum_{k}\left|V_{k, \sigma}\right|^{2} \delta\left(\omega-\varepsilon_{k, \sigma}\right)$. For the impurity model, where we are interested in universal features, it is usual to take a wide conduction band with a flat density of states so that $\Delta_{\sigma}(\omega)$ becomes independent of $\omega$, and can be taken as a constant $\Delta_{\sigma}$. In this wide band limit $\Delta_{\sigma}(\omega)$ will be independent of the magnetic field on the conduction electrons, so we can effectively put $g_{c}=0$. When this is the case $\Delta_{\sigma}$ is usually taken to be a constant $\Delta$ independent of $\sigma$.

In the renormalized perturbation theory approach ${ }^{6.7}$ we cast the corresponding Lagrangian for this model 


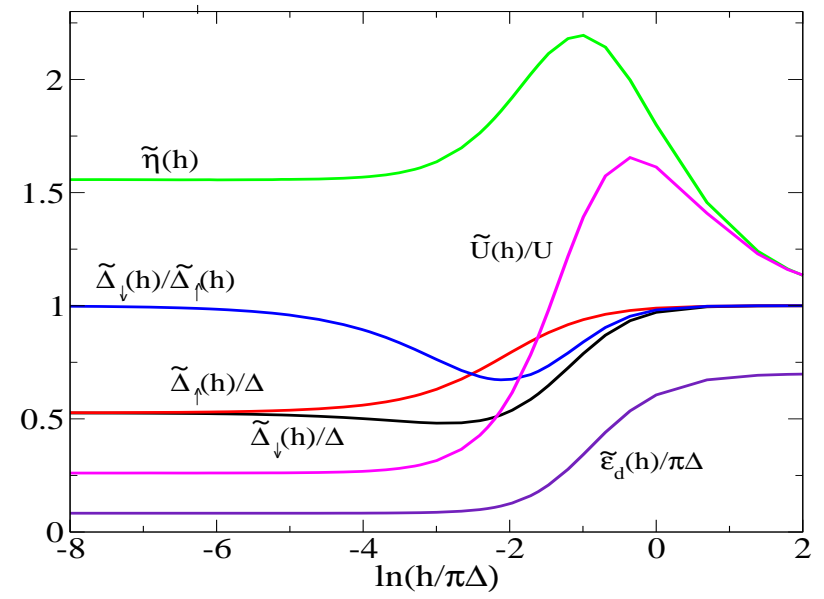

FIG. 1: (Color online) Plots of the renormalized parameters, $\tilde{\Delta}_{\uparrow}(h) / \Delta, \tilde{\Delta}_{\downarrow}(h) / \Delta, \tilde{\varepsilon}_{\mathrm{d}}(h) / \pi \Delta, \tilde{U}(h) / U, \tilde{\eta}(h)$, for the asymmetric Anderson model, with $\pi \Delta=0.1, U / \pi \Delta=2$ and $\varepsilon_{d} / \pi \Delta=-0.3$, as a function of the logarithm of the magnetic field $h / \pi \Delta$. The ratio $\tilde{\Delta}_{\downarrow}(h) / \tilde{\Delta}_{\uparrow}(h)$ is also shown.

$\mathcal{L}_{\mathrm{AM}}\left(\varepsilon_{\mathrm{d}, \sigma}, \Delta, U\right)$ into the form,

$$
\mathcal{L}_{\mathrm{AM}}\left(\varepsilon_{\mathrm{d}, \sigma}, \Delta, U\right)=\mathcal{L}_{\mathrm{AM}}\left(\tilde{\varepsilon}_{\mathrm{d}, \sigma}, \tilde{\Delta}_{\sigma}, \tilde{U}\right)+\mathcal{L}_{\mathrm{ct}}\left(\lambda_{1}, \lambda_{2}, \lambda_{3}\right),
$$

where the renormalized parameters, $\tilde{\varepsilon}_{\mathrm{d}, \sigma}$ and $\tilde{\Delta}_{\sigma}$, are defined in terms of the self-energy $\Sigma_{\sigma}(\omega)$ of the one-electron Green function for the impurity state,

$$
G_{\sigma}(\omega)=\frac{1}{\omega-\varepsilon_{\mathrm{d} \sigma}+i \Delta-\Sigma_{\sigma}(\omega)},
$$

and are given by

$$
\tilde{\varepsilon}_{\mathrm{d}, \sigma}=z_{\sigma}\left(\varepsilon_{\mathrm{d}, \sigma}+\Sigma_{\sigma}(0)\right), \quad \tilde{\Delta}_{\sigma}=z_{\sigma} \Delta,
$$

where $z_{\sigma}$ is given by $z_{\sigma}=1 /\left(1-\Sigma_{\sigma}^{\prime}(0)\right)$. The renormalized or quasiparticle interaction $\tilde{U}$, is defined in terms of the local total 4 -vertex $\Gamma_{\uparrow \downarrow}\left(\omega_{1}, \omega_{2}, \omega_{3}, \omega_{4}\right)$ at zero frequency,

$$
\tilde{U}=z_{\uparrow} z_{\downarrow} \Gamma_{\uparrow \downarrow}(0,0,0,0) .
$$

It will be convenient to rewrite the spin dependent quasiparticle energies in the form, $\tilde{\varepsilon}_{\mathrm{d}, \sigma}=\tilde{\varepsilon}_{\mathrm{d}}(h)-\sigma h \tilde{\eta}(h)$, where

$$
\tilde{\varepsilon}_{\mathrm{d}}(h)=\frac{1}{2} \sum_{\sigma} \tilde{\varepsilon}_{\mathrm{d}, \sigma}, \quad \tilde{\eta}(h)=\frac{1}{2 h} \sum_{\sigma} \sigma \tilde{\varepsilon}_{\mathrm{d}, \sigma}
$$

where $\tilde{\varepsilon}_{\mathrm{d}}(h)$ and $\tilde{\eta}(h)$ are both even functions of the magnetic field $h=g \mu_{\mathrm{B}} H / 2$.

The renormalized perturbation expansion is in powers of the renormalized interaction $U$ for the complete Lagrangian defined in equation (2). The counter term part of the Lagrangian $\mathcal{L}_{\mathrm{ct}}\left(\lambda_{1}, \lambda_{2}, \lambda_{3}\right)$ essentially takes care of any overcounting. The parameters, $\tilde{\varepsilon}_{\mathrm{d}, \sigma}, \tilde{\Delta}_{\sigma}$ and $\tilde{U}$, have been taken to be the fully renormalized ones.
The most effective way of estimating them in this case is from the energy levels of a numerical renormalization group (NRG) calculation ${ }^{3.5}$. However, the approach itself is independent of the NRG, and the parameters can be deduced in other ways, for instance from experiment. The counter term parameters, $\lambda_{1}, \lambda_{2}$ and $\lambda_{3}$, are required to cancel any further renormalization, and are completely determined by this condition ${ }^{6.7}$.

In figure 1 we display some typical results for renormalized parameters as a function of the magnetic field on a $\log$ scale. For this plot we have taken bare parameters $\varepsilon_{\mathrm{d}} / \pi \Delta=-0.3$ and $U / \pi \Delta=2$, corresponding to a impurity occupation in the absence of a field, $\left\langle n_{d, \sigma}\right\rangle=n(0) / 2 \sim 0.35$. With these values the parameters are strongly renormalized for $h=0$. The overall trend as a function of $h$ is very similar to that for the particle-hole symmetric case (I) in the strong coupling regime, with the parameters converging to the mean field values in the limit $h \rightarrow \infty$. We do see, however, that the resonance widths become spin dependent, $\Delta_{\uparrow}(h) \neq \Delta_{\downarrow}(h)$, except asymptotically as $h \rightarrow 0$ and $h \rightarrow \infty$. We note that, though $\tilde{\Delta}_{\uparrow}(h)$ increases monotonically with increase of $h, \tilde{\Delta}_{\downarrow}(h)$ initially decreases. In this case, where the impurity level is less than half-filled, the ratio $\tilde{\Delta}_{\uparrow}(h) / \tilde{\Delta}_{\downarrow}(h) \geq 1$. This ratio is reversed, so $\tilde{\Delta}_{\uparrow}(h) / \tilde{\Delta}_{\downarrow}(h) \leq 1$, when the impurity level is more than half-filled.

\section{STATIC RESPONSE FUNCTIONS}

Once the renormalized parameters have been determined, the static impurity response functions can be evaluated directly by substituting into the relevant $e x$ act formula. This is a more direct way of calculating these response functions than the usual NRG way, which involves a subtraction procedure to estimate the impurity component $10,11,12$. The formulae in the absence of a magnetic field ${ }^{3}$, and for the symmetric model in the presence of a field, were given earlier (I). Here, we give the generalizations for the non-symmetric model. The induced magnetization $M(h)$ is given by $M(h)=g \mu_{\mathrm{B}} m(h)$, where

$$
m(h)=\frac{1}{2}\left(n_{\mathrm{d} \uparrow}-n_{\mathrm{d} \downarrow}\right)=\frac{-1}{2 \pi} \sum_{\sigma} \sigma \tan ^{-1}\left(\frac{\tilde{\varepsilon}_{\mathrm{d} \sigma}(h)}{\tilde{\Delta}_{\sigma}(h)}\right),
$$

which can be derived from the Friedel sum rule. The longitudinal susceptibility $\chi_{l}(h)$ (in units of $\left.\left(g \mu_{\mathrm{B}}\right)^{2}\right)$ is given by

$$
\chi_{l}(h)=0.25\left(\tilde{\rho}_{\uparrow}(0, h)+\tilde{\rho}_{\downarrow}(0, h)+\tilde{U}(h) \tilde{\rho}_{\uparrow}(0, h) \tilde{\rho}_{\downarrow}(0, h)\right),
$$

where $\tilde{\rho}_{\sigma}(\omega, h)$ is the free quasiparticle density of states given by

$$
\tilde{\rho}_{\sigma}(\omega, h)=\frac{1}{\pi} \frac{\tilde{\Delta}_{\sigma}(h)}{\left(\omega-\tilde{\varepsilon}_{\mathrm{d}, \sigma}(h)\right)^{2}+\tilde{\Delta}_{\sigma}^{2}(h)} .
$$




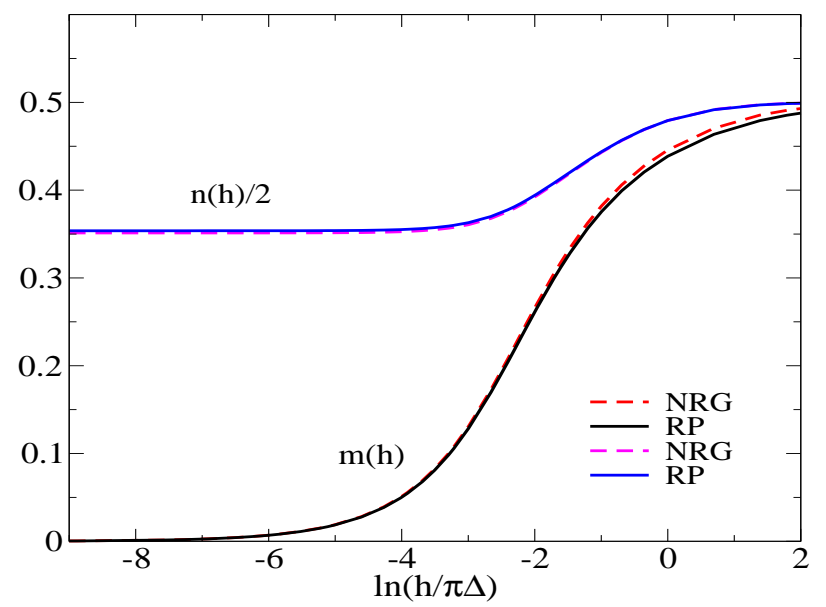

FIG. 2: (Color online) The induced magnetization $m(h)$ as a function of the logarithm of the magnetic field $h$ for the asymmetric Anderson model with the same set of parameters as given in figure 1. The dashed curve is that calculated from the direct evaluation of the occupation values from the NRG ground state, and the full curve is that deduced from the renormalized parameters in equation (7). Also shown is the average occupation $n(h) / 2$ as calculated from the NRG ground state (dashed curve) and the quasiparticle occupation values (full curve) as given in equation (11).

The corresponding transverse susceptibility $\chi_{t}(h)$ (zero applied field limit in the transverse direction) is given by

$$
\chi_{t}(h)=\frac{m(h)}{2 h} .
$$

The total occupation of the impurity site $n(h)=\left(n_{\mathrm{d} \uparrow}+\right.$ $\left.n_{\mathrm{d} \downarrow}\right)$ can be derived similarly, and is given by

$$
n(h)=1-\frac{1}{2 \pi} \sum_{\sigma} \tan ^{-1}\left(\frac{\tilde{\varepsilon}_{\mathrm{d} \sigma}(h)}{\tilde{\Delta}_{\sigma}(h)}\right),
$$

and the local charge susceptibility $\chi_{c}(h)$ is given by

$$
\chi_{c}(h)=0.25\left[\tilde{\rho}_{\uparrow}(0, h)+\tilde{\rho}_{\downarrow}(0, h)-\tilde{U}(h) \tilde{\rho}_{\uparrow}(0, h) \tilde{\rho}_{\downarrow}(0, h)\right] .
$$

To illustrate the magnetic field dependence of these static response functions, we evaluate these formulae in a particular case using the field dependent renormalized parameters given in figure 1. The results are compared with those obtained by a evaluation of static expectation values using the NRG. In all subsequent calculations in this paper, we concentrate on this same set of bare parameters, $\varepsilon_{\mathrm{d}} / \pi \Delta=-0.3$ and $U / \pi \Delta=2$.

Shown in figure 2 is the result for $m(h)$ deduced from equation (7), compared with results obtained by the direct evaluation of the d-site occupation values in the ground state as determined from the NRG. There is a small but systematic difference, of the order of $2 \%$, between the two sets of results. This difference could be due to the fact that we assume an infinite bandwidth in the derivation of these formulae, whereas we take a

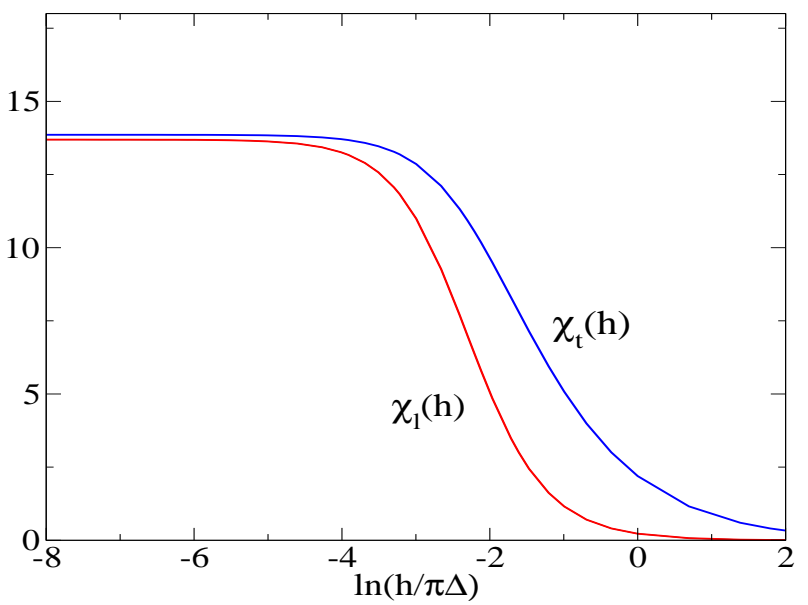

FIG. 3: (Color online) The longitudinal and transverse impurity site susceptibilities, $\chi_{l}(h)$ and $\chi_{t}(h)$, as a function of the logarithm of the magnetic field $h . \chi_{l}(h)$ is calculated from equation (8) and $\chi_{t}(h)=m(h) / 2 h$.

finite band width $2 D$, with $D=1$ in the NRG calculations. The corresponding estimates of the average occupation number $n(h) / 2$ as a function of magnetic field $h$ are shown in the same figure. For this quantity the two sets of results are almost indistinguishable. In the extreme large field limit the average occupation of the impurity level tends to unity, as the majority spin level gets pulled further and further below the Fermi level, and the impurity becomes completely polarized. In this regime the average renormalized level, $\tilde{\varepsilon}_{\mathrm{d}}$, approaches the mean field value, $\tilde{\varepsilon}_{\mathrm{d}}=\varepsilon_{\mathrm{d}}+0.5 U n(h)$, rather than the bare value $\varepsilon_{\mathrm{d}}$, while the other renormalized quantities approach their bare values.

The longitudinal and transverse spin susceptibilities, $\chi_{l}(h)$ and $\chi_{t}(h)$, are plotted in figure 3 as a function of the logarithm of the magnetic field $h . \chi_{l}(h)$ is calculated from equation (8) and $\chi_{t}(h)=m(h) / 2 h$ as calculated from equation (77). They should asymptotically converge to the same result in the limit $h \rightarrow 0$. There seems to be a very small discrepancy, of about $1 \%$, between the two estimates in this limit. This could be due to evaluating the ratio in equation (10) for very small values of the field.

We now look at the behavior of the corresponding dynamic response functions.

\section{DYNAMIC RESPONSE FUNCTIONS}

\section{A. Single particle spectra}

It is of interest to see how well the free quasiparticle density of states given in equation (9) compares with the spectral density $\rho_{\sigma}(\omega)$ calculated from the NRG for each spin type in the presence of a field. The NRG spectra are calculated from the self-energy follow- 

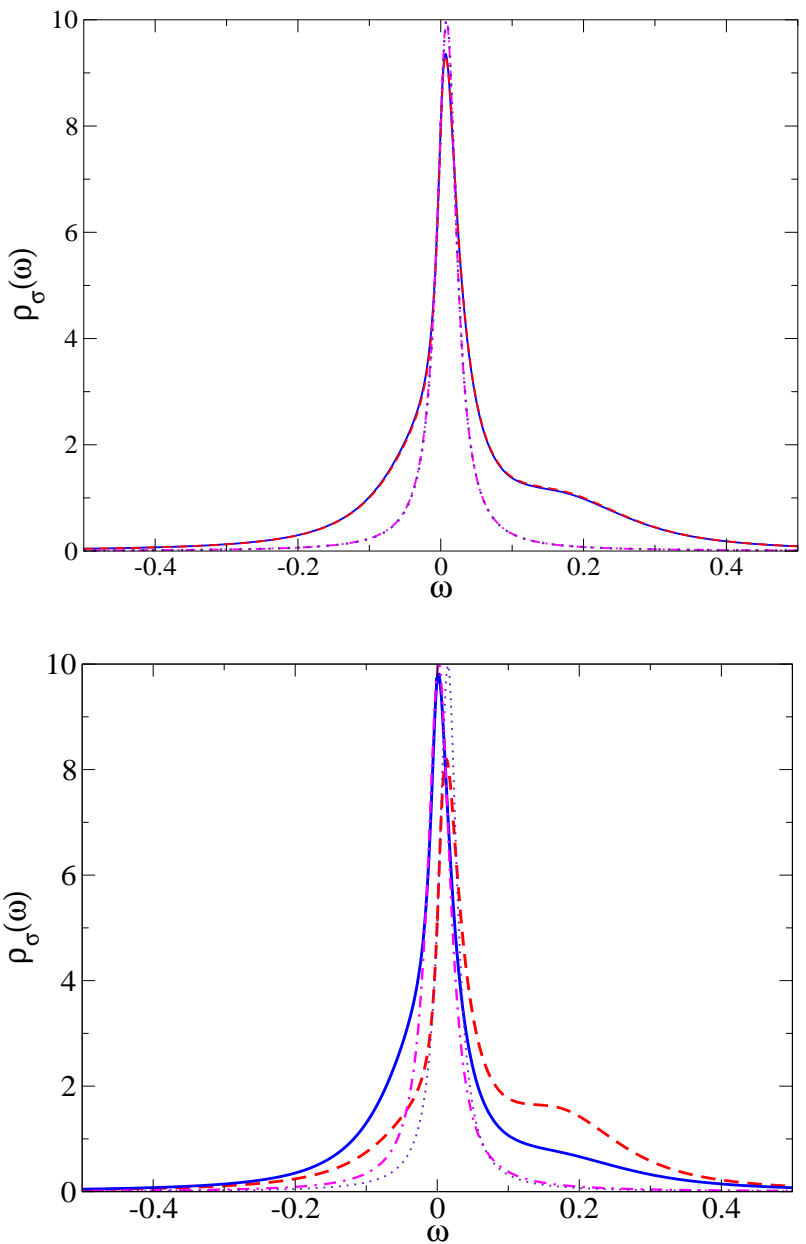

FIG. 4: (Color online) The spectral density for the spin up (majority) electrons $\rho_{\uparrow}(\omega, h)$ (full curve) and spin down (minority) electrons $\rho_{\downarrow}(\omega, h)$ (dashed curve) at $T=0$ as a function of $\omega$ for $h / \pi \Delta=0.001$ (upper panel) and $h / \pi \Delta=0.04$ (lower panel). The two curves on the upper panel are almost coincident on the scale shown. Also shown are the corresponding quantities derived from the free quasiparticle densities of states, $z_{\uparrow} \tilde{\rho}_{\uparrow}(\omega)$ (dot-dashed) and $z_{\downarrow} \tilde{\rho}_{\downarrow}(\omega)$ (dotted).

ing the prescription given in $\frac{13}{3}$, and we have also used the improved method $\underline{14,15}$ based on the complete AndersSchiller basis 16 .

In figure 4 (upper panel) we show $\rho_{\uparrow}(\omega)$ and $\rho_{\downarrow}(\omega)$, as calculated from the NRG, for the field $h / \pi \Delta=0.001$.

We see that in this relatively weak field that there are only a small differences between the two spectral densities; they are almost coincident on the scale shown. The corresponding quantities derived from the free quasiparticle densities of states, $z_{\uparrow} \tilde{\rho}_{\uparrow}(\omega)$ and $z_{\downarrow} \tilde{\rho}_{\downarrow}(\omega)$, are shown in the same figure.

In figure 4 (lower panel) we make a similar comparison for a large field $h / \pi \Delta=0.04$, and the same set of bare parameters. The polarization is much stronger for this higher magnetic field value and there is now a marked difference between the two spectral densities for the two spin types. The spectra derived from the free quasiparti-

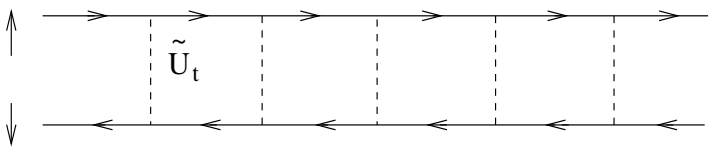

FIG. 5: Repeated scattering of a quasiparticle with spin $\uparrow$ and a quasihole with spin $\downarrow$ via the effective interaction $\tilde{U}_{t}$.

cles, can be seen to describe the spectrum in the immediate vicinity of the Fermi level for both spin types. They cannot describe the difference in the heights of the peaks of the two spin types, because in these simple Lorentzian formula the height of peaks in the quasiparticle weighted spectra are independent of $H$ and the spin and given by $1 / \pi \Delta$. Note that the description of the low frequency range can be extended within the renormalized perturbation theory frame work by including a self-energy correction in the quasiparticle density of states as shown in the particle hole symmetric case $\frac{17}{}$.

In the next section we look beyond the simple free quasiparticle picture and take account of the leading quasiparticle scattering terms.

\section{B. Dynamic spin susceptibilities}

The leading corrections to the two particle dynamic response functions to the free quasiparticle picture in the renormalized perturbation theory arise from the diagrams with repeated quasiparticle scattering. In figure 5 we illustrate this type of diagram for the scattering of a free up spin quasiparticle with a free down spin quasihole.

Such a diagram contributes to the transverse dynamic susceptibility $\chi_{t}(\omega, h)$ in the presence of the magnetic field. We derived a quasiparticle interaction term $\tilde{U}(h)$ earlier, but this is not the interaction term we need in considering this diagram, as this corresponds to the total 4-vertex, $\tilde{\Gamma}_{\uparrow \downarrow}(0,0,0,0)$, which has implicitly included this scattering for $\omega=0$. What we use is the irreducible particle-hole interaction at zero frequency in this channel, which we denote by $\tilde{U}_{t}(h)$. The dynamic transverse susceptibility $\chi_{t}(\omega, h)$, is then given by

$$
\chi_{t}(\omega, h)=\frac{\tilde{\chi}_{\uparrow \downarrow}(\omega, h)}{1-\tilde{U}_{t}(h) \tilde{\chi}_{\uparrow \downarrow}(\omega, h)},
$$

where $\tilde{\chi}_{\uparrow \downarrow}(\omega, h)$ is the transverse spin susceptibility of the free quasiparticle. The analytic expression for $\tilde{\chi}_{\uparrow \downarrow}(\omega, h)$ is given in the appendix.

To find the unknown quantity $\tilde{U}_{t}(h)$ we use the fact that this series for $\omega=0$ gives the static transverse susceptibility which we have calculated already, and is given in equation (10). Hence, comparing (13) for $\omega=0$ with 
equation (10) yields

$$
\tilde{U}_{t}(h)=\frac{1}{m(h)}\left\{\frac{4 \tilde{h}^{2}+\left(\tilde{\Delta}_{\uparrow}-\tilde{\Delta}_{\downarrow}\right)^{2}}{4 \tilde{h}+\frac{\left(\tilde{\Delta}_{\uparrow}-\tilde{\Delta}_{\downarrow}\right)}{2 \pi m(h)} \ln \left(\frac{\left(\tilde{h}-\tilde{\varepsilon}_{d}\right)^{2}+\tilde{\Delta}_{\uparrow}^{2}}{\left(\tilde{h}+\tilde{\varepsilon}_{d}\right)^{2}+\tilde{\Delta}_{\downarrow}^{2}}\right)}-h\right\},
$$

where $\tilde{h}=h \tilde{\eta}(h)$, and $m(h)$ is given in equation (7). This expression simplifies in the case, $\tilde{\Delta}_{\uparrow}=\tilde{\Delta}_{\downarrow}$, to give $\tilde{U}_{t}(h)=(\tilde{h}-h) / m(h)$, which is the same as that used earlier ${ }^{8}$.

Now we test how well these formulae describe the spin dynamic response over the relevant frequency range. To do this we compare the predictions based on the RPT formula (13) with those obtained from a direct NRG evaluation.

The imaginary part of the transverse dynamic susceptibility is shown in figure 6 (upper panel) for a magnetic field value $h / \pi \Delta=0.001$. The dashed curve is that from a direct $\mathrm{NRG}$ evaluation ${ }^{18,19}$ and the full curve is that calculated using equation (13), with the corresponding renormalized parameters. There is very good agreement between the two sets of results.

Results for the imaginary part of $\chi_{t}(\omega, h)$ for the field value $h / \pi \Delta=0.04$ are shown in figure 6 (lower panel). In this stronger field one of the peaks is suppressed while the other peak is enhanced. The peak positions are in good agreement, and the slightly broader peak from the NRG data can be attributed to the logarithmic broadening used in the direct NRG evaluation. There is a sum rule, that the total spectral weight is equal to $-2 m(h)$, which is satisfied precisely both in the RPT result and also in the NRG calculation, as we have used the improved prescription for the response functions based on the complete Anders-Schiller basis.

In a similar way, we can calculate the longitudinal dynamic susceptibility $\chi_{l}(\omega, h)$ from repeated scattering of the spin up and spin down quasiparticles. The irreducible zero frequency interaction in this channel we denote by $\tilde{U}_{l}(h)$, and determine it from the condition, $\chi_{l}(0, h)=\chi_{l}(h)$, using equation (8). The results for $\chi_{l}(\omega, h)$ is

$$
\begin{aligned}
& \chi_{l}(\omega, h)= \\
& \frac{\tilde{\chi}_{\uparrow \uparrow}(\omega, h)+\tilde{\chi}_{\downarrow \downarrow}(\omega, h)+4 \tilde{U}_{l}(h) \tilde{\chi}_{\uparrow \uparrow}(\omega, h) \tilde{\chi}_{\downarrow \downarrow}(\omega, h)}{2\left(1-4 \tilde{U}_{l}^{2}(h) \tilde{\chi}_{\uparrow \uparrow}(\omega, h) \tilde{\chi}_{\downarrow \downarrow}(\omega, h)\right)},
\end{aligned}
$$

where the analytic expression for $\tilde{\chi}_{\sigma \sigma}(\omega, h)$ is given in the Appendix. Equation (15) is a generalization to our earlier result ${ }^{8}$ for the particle-hole symmetric model. The zero frequency irreducible particle-hole vertex $\tilde{U}_{l}(h)$ in this scattering channel is given by

$$
\tilde{U}_{l}(h)=\frac{-1+\sqrt{\left[1+\tilde{U}^{2}(h)\left(\tilde{\rho}_{\uparrow}+\tilde{\rho}_{\downarrow}\right)\right]^{2}-\tilde{U}^{2}(h)\left(\tilde{\rho}_{\uparrow}-\tilde{\rho}_{\downarrow}\right)^{2}}}{2\left(\tilde{\rho}_{\uparrow}+\tilde{\rho}_{\downarrow}+\tilde{U}(h) \tilde{\rho}_{\uparrow} \tilde{\rho}_{\downarrow}\right)},
$$

where we have simplified the notation, $\tilde{\rho}_{\sigma}(0, h)=\tilde{\rho}_{\sigma}$. In the absence of a magnetic field, or with a magnetic field
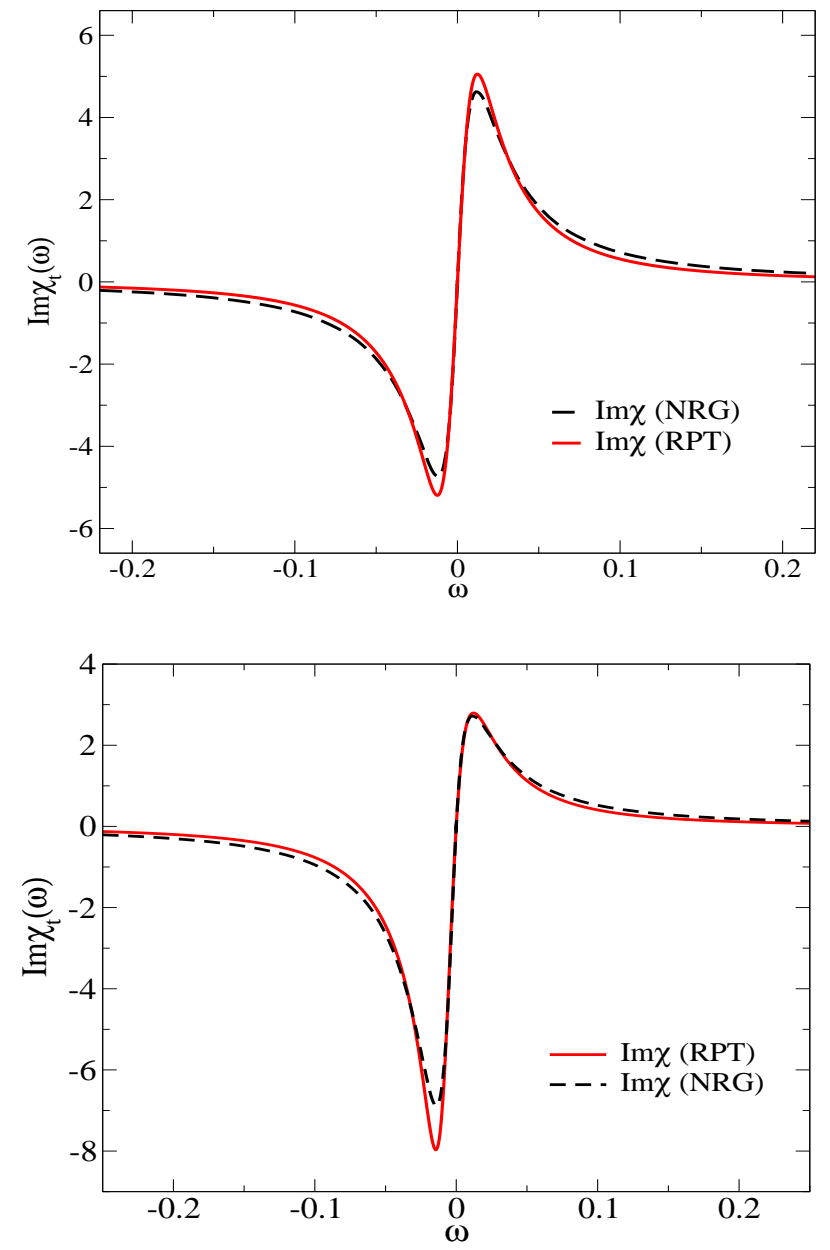

FIG. 6: (Color online) The imaginary part of the transverse dynamic susceptibility, $\chi_{t}(\omega, h)$ at $T=0$ as a function of $\omega$, for $h / \pi \Delta=0.001$ (upper panel) and $h / \pi \Delta=0.04$ (lower panel). The dashed curve is calculated from a direct NRG calculation and the full curve from equation (13) with the renormalized parameters.

for the particle-hole symmetric model, $\tilde{\rho}_{\uparrow}=\tilde{\rho}_{\downarrow}$, the result simplifies to $\tilde{U}_{l}(h)=\tilde{U}(h) /[1+\tilde{U}(h) \tilde{\rho}(0, h)]$, which is the value used in the earlier work ${ }^{8}$.

The imaginary part of the longitudinal dynamic spin susceptibility in the weak field case, $h / \pi \Delta=0.001$, is similar to that for the transverse case shown in figure 6 (upper panel), apart from an overall factor of 2 , so we do not show he results here. The stronger field case, $h / \pi \Delta=0.04$, is shown in figure 7 .

Due to the stronger magnetic field, the heights of the peaks are slightly reduced as compared with the results for $h / \pi \Delta=0.001$, but the overall features are very similar. The Korringa-Shiba relation does not hold for the model without particle-hole symmetry in the presence of a magnetic field. Where it does hold, in the absence of a field, or with particle-hole symmetry, the renormalized perturbation expression satisfies it exactly ${ }^{8}$. 


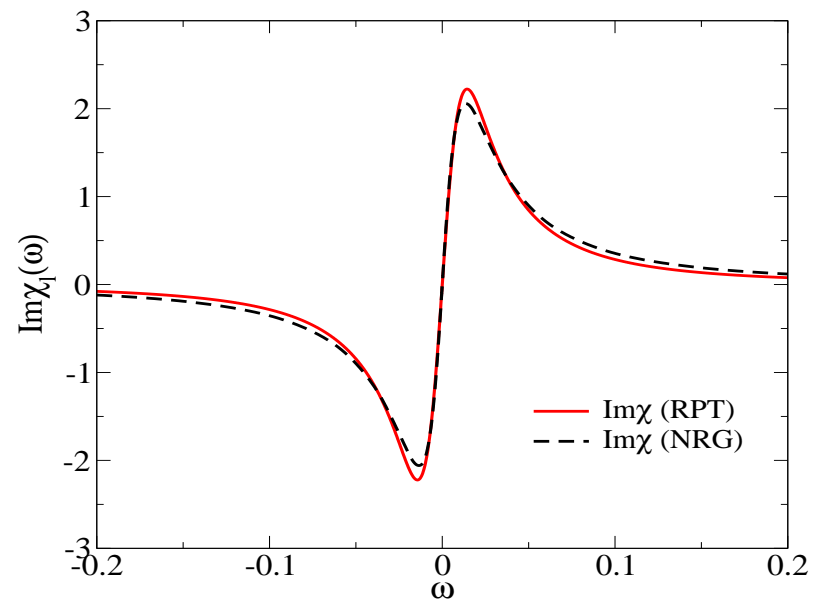

FIG. 7: (Color online) The imaginary part of the longitudinal dynamic susceptibility, $\chi_{l}(\omega, h)$ at $T=0$ as a function of $\omega$ for $(h / \pi \Delta=0.04)$.

\section{SUMMARY AND DISCUSSION}

In this paper we have extended our earlier work I, where we described the low energy behavior of the symmetric Anderson model in a magnetic field $h$ in terms of field-dependent renormalized quasiparticles, to the nonsymmetric Anderson model. The main new feature that emerges is the dependence of the quasiparticle peak resonance width $\tilde{\Delta}_{\sigma}(h)$ on the spin type $\sigma$ as well as on the value of the magnetic field. The $T=0$ spin and charge susceptibilities can be expressed as exact formulae in terms of the parameters $\Delta_{\sigma}(h), \tilde{\varepsilon}_{\mathrm{d}, \sigma}(h)$ and a local field dependent interaction between the quasiparticles $\tilde{U}(h)$. Based on these parameters the low temperature behavior of the model, such as the susceptibilities and conductance, in the presence of the field could be calculated as described for the symmetric model in I. It was also demonstrated earlier that an excellent description of the low energy spin dynamics can be obtained for the symmetric mode ${ }^{18}$ based on approximate formulae which take into account repeated quasiparticle scattering in the RPT. Here these results have been generalized to the nonsymmetric model for the transverse and longitudinal spin susceptibilities, which again agree remarkably well with those obtained from a direct NRG calculation.

What may seem surprising at first is that the results for the dynamic susceptibilities, based on the repeated quasiparticle scattering, agree so well over the full range of $\omega$, whereas the free quasiparticle density of states only describes the single electron spectral densities well only in the immediate region of the Fermi level. However, as discussed in I, the quasiparticle density of states $\tilde{\rho}_{\sigma}(\omega)$, survives in the limit $z_{\sigma} \rightarrow 0$, when it becomes a delta function describing a free spin. For very small $z_{\sigma}$ the product $z_{\sigma} \tilde{\rho}_{\sigma}(\omega)$, makes very little contribution to the spec- tral density $\rho_{\sigma}(\omega)$, except near the Fermi level. Hence in the strong correlation regime, when $z_{\sigma}$ is very small, it would appear to be more appropriate to interpret the quasiparticle as describing a spinon excitation. Because the dominant low energy excitations are spinons, as is known from the Bethe ansatz solutions for the Kondo model ${ }^{20,21}$, then it is not so surprising that they provide a good description of the spin dynamics.

\section{Acknowledgement}

We wish to thank N. Dupuis, D.M. Edwards, W. Koller, D. Meyer and A. Oguri for helpful discussions and W. Koller and D. Meyer for their contributions to the development of the NRG programs. One of us (J.B.) thanks the Gottlieb Daimler and Karl Benz Foundation, the German Academic exchange service (DAAD) and the EPSRC for financial support.

\section{APPENDIX}

The free quasiparticle dynamic susceptibility $\tilde{\chi}_{\sigma, \sigma^{\prime}}(\omega)$ for the impurity model in the wide band limit, $\tilde{\Delta}_{\uparrow}=\tilde{\Delta}_{\downarrow}$, were given earlier ${ }^{8}$. Here we give the more general results for $\tilde{\Delta}_{\uparrow} \neq \tilde{\Delta}_{\downarrow}$,

$$
\tilde{\chi}_{\sigma, \sigma}(\omega)=\frac{-1}{\pi \omega} \frac{\tilde{\Delta}_{\sigma}}{\omega-2 i \tilde{\Delta}_{\sigma}} \sum_{\alpha=-1,1} \ln \left(1-\frac{\omega}{\alpha \tilde{\varepsilon}_{d, \sigma}+i \tilde{\Delta}_{\sigma}}\right),
$$

for $\omega>0$, and for $\omega=0$,

$$
\tilde{\chi}_{\sigma, \sigma}(0)=\tilde{\rho}_{\sigma}(0) .
$$

The values for $\omega<0$ follow from the fact that $\operatorname{Re} \tilde{\chi}_{\sigma, \sigma}(\omega)=\operatorname{Re} \tilde{\chi}_{\sigma, \sigma}(-\omega)$ and $\operatorname{Im} \tilde{\chi}_{\sigma, \sigma}(\omega)=$ $-\operatorname{Im} \tilde{\chi}_{\sigma, \sigma}(-\omega)$. For $\sigma^{\prime} \neq \sigma$,

$$
\begin{aligned}
& \tilde{\chi}_{\uparrow, \downarrow}(\omega)= \\
& \frac{i / 2 \pi}{\left(\omega+\tilde{\varepsilon}_{d, \downarrow}-\tilde{\varepsilon}_{d, \uparrow}+i \tilde{\Delta}_{\uparrow}-i \tilde{\Delta}_{\downarrow}\right)} \ln \left(\frac{\omega-\tilde{\varepsilon}_{d, \uparrow}-i \tilde{\Delta}_{\uparrow}}{-i \tilde{\Delta}_{\downarrow}-\tilde{\varepsilon}_{d, \downarrow}}\right) \\
& +\frac{i / 2 \pi}{\left(\omega+\tilde{\varepsilon}_{d, \downarrow}-\tilde{\varepsilon}_{d, \uparrow}-i \tilde{\Delta}_{\uparrow}+i \tilde{\Delta}_{\downarrow}\right)} \ln \left(\frac{\omega+\tilde{\varepsilon}_{d, \downarrow}-i \tilde{\Delta}_{\downarrow}}{-i \tilde{\Delta}_{\uparrow}+\tilde{\varepsilon}_{d, \uparrow}}\right) \\
& +\frac{-i / 2 \pi}{\left(\omega+\tilde{\varepsilon}_{d, \downarrow}-\tilde{\varepsilon}_{d, \uparrow}+i \tilde{\Delta}_{\uparrow}+i \tilde{\Delta}_{\downarrow}\right)} \times \\
& \times\left[\ln \left(1+\frac{\omega}{i \tilde{\Delta}_{\uparrow}-\tilde{\varepsilon}_{d, \uparrow}}\right)+\ln \left(1+\frac{\omega}{i \tilde{\Delta}_{\downarrow}+\tilde{\varepsilon}_{d, \downarrow}}\right)\right] .
\end{aligned}
$$

${ }^{1}$ A. C. Hewson, J. Bauer, and W. Koller, Phys. Rev. B 73, 045117 (2006).
${ }^{2}$ P. W. Anderson, Phys. Rev. 124, 41 (1961). 
3 A. C. Hewson, A. Oguri, and D. Meyer, Eur. Phys. J. B 40, 177 (2004).

4 A. C. Hewson, The Kondo Problem to Heavy Fermions (Cambridge University Press, Cambridge, 1993).

5 A. C. Hewson, J. Phys. Soc. Japan 74, 8 (2005).

6 A. C. Hewson, Phys. Rev. Lett. 70, 4007 (1993).

7 A. C. Hewson, J. Phys.: Cond. Mat. 13, 10011 (2001).

8 A. C. Hewson, J. Phys.: Cond. Mat. 18, 1815 (2006).

${ }^{9}$ H. Shiba, Prog. Theor. Phys. 54, 967 (1975).

${ }^{10}$ K. Wilson, Rev. Mod. Phys. 47, 773 (1975).

${ }^{11}$ H. R. Krishna-murthy, J. W. Wilkins, and K. G. Wilson, Phys. Rev. B 21, 1003 (1980).

12 H. R. Krishna-murthy, J. W. Wilkins, and K. G. Wilson, Phys. Rev. B 21, 1044 (1980).

13 R. Bulla, A. C. Hewson, and T. Pruschke, J. Phys.: Cond. Mat. 10, 8365 (1998).

14 R. Peters, T. Pruschke, and F. B. Anders, Phys. Rev. B
74, 245114 (2006).

15 A. Weichselbaum and J. von Delft (2006), condmat/0607497.

16 F. B. Anders and A. Schiller, Phys. Rev. Lett. 95, 196801 (2005).

17 J. Bauer, A. C. Hewson, and A. Oguri, J. Magn. Magn. Mat. 310, 1133 (2007).

18 O. Sakai, Y. Shimizu, and T. Kasuya, J. Phys. Soc. Japan 58, 3666 (1989).

19 T. A. Costi, A. C. Hewson, and V. Zlatić, J. Phys.: Cond. Mat. 6, 2519 (1994).

20 A. M. Tsvelik and P. B. Wiegmann, Adv. Phys. 32, 453 (1983).

21 N. Andrei, K. Furuya, and J. H. Lowenstein, Rev. Mod. Phys. 55, 331 (1983). 